\title{
Análisis de las modificaciones en el índice de masa corporal en un grupo de pacientes con obesidad y mayores de edad'
}

Raquel Alba Martín²

Juan Luis Salceda García ${ }^{3}$

doi: 10.11 144/Javeriana.ie18-2.amim

Cómo citar: Martín RA, Salceda García JL. Análisis de las modificaciones en el índice de masa corporal en un grupo de pacientes con obesidad y mayores de edad. Investig Enferm. Imagen Desarr. 2016;18(2): 31-42. http://dx.doi.org/10.11144/Javeriana.ie18-2.amim

1. Artículo original de investigación. Fecha de recepción: 16 de mayo de 2015. Fecha de aceptación: 1 de diciembre de 2015.

2. Máster en técnico Superior en Prevención de Riesgos Laborales. Experta Universitaria en Investigación Enfermera, Fuden, Madrid, España. Correo electrónico: raquelalbamartin@, satse.es

3. Técnico en transporte sanitario. Correo electrónico: julusalgar@gmail.com 


\section{Resumen}

Objetivo: Analizar los cambios en el indice de masa corporal (IMC) en un grupo de pacientes obesos tras una intervención enfermera basada en promover cambios en su estilo de vida, mediante dieta hipocalórica, ejercicio físico y terapia conductual. Método: Estudio cuasi experimental con un muestreo no probabilístico de 32 pacientes cuyos criterios de inclusión fueron pacientes sanos, con autonomía para las actividades de la vida diaria y sin demencia senil. La intervención se fundamentó en una intervención enfermera basada en promover cambios en su estilo de vida y, posteriormente, se evaluó a través del descenso en el IMC. Resultados: El valor medio del IMC basal era de 34,2 al comienzo del estudio. Tras la intervención enfermera existe una progresiva disminución del IMC que fue estadísticamente significativa a los 6 meses de tratamiento, que resultó un valor medio en el IMC de 32,7. Es decir, una reducción del IMC en torno al 4\%. Conclusiones: La intervención enfermera personalizada y el seguimiento intensivo favorecen una buena aceptación o adhesión al programa y permiten observar cambios en el estilo de vida de los pacientes imprescindibles para el control de la obesidad.

Palabras clave: obesidad; educación para la salud; enfermería; adultez

\section{Analysis of Body Mass Index Changes in a Group of Adult Patients with Obesity}

\section{Abstract}

Objective: To analyze changes in body mass index (BMI) in a group of obese patients after a nurse-based intervention to promote changes in their lifestyle through lowcalorie diet, exercise and behavioral therapy. Method: quasi-experimental study with a non-probabilistic sample of 32 patients whose inclusion criteria were healthy patients, with autonomy for activities of daily living without dementia. The intervention was based on a nurse-based intervention to promote changes in their lifestyle and was subsequently evaluated by the decrease in BMI. Results: The mean value of the BMR was 34, 2 at the beginning of the study. After the nursing intervention there is a progressive decrease in BMI which was statistically significant after 6 months of treatment, which resulted in an mean value of 32,7. That is, a reduction in BMI of around $4 \%$. Conclusions: personalized nursing intervention and intensive follow-up favor good acceptance or accession to the program and allow us to observe changes in the lifestyle of patients which is essential for controlling obesity.

Keywords: obesity; education for health; Nursing; adulthood 


\section{Análise das alterações no índice de massa corporal em uma turma de pacientes com obesidade e idosos}

\section{Resumo}

Objetivo: Analisar as mudanças no índice de massa corporal (IMC) em uma turma de pacientes obesos após intervenção de enfermagem baseada em promover mudanças no seu estilo de vida, mediante dieta hipocalórica, exercício físico e terapia condutual. Método: Estudo quase-experimental com amostragem não probabilístico de 32 pacientes cujos critérios de inclusão foram pacientes saudáveis, com autonomia para as atividades da vida diária e sem demência senil. A intervenção fundamentou-se em intervenção de enfermagem baseada em promover mudanças no estilo de vida e, posteriormente, foi avaliado através do descenso no IMC. Resultados: O valor médio do IMC basal era de 34,2 no começo do estudo. Após a intervenção de enfermagem há uma progressiva diminuição do IMC estatisticamente significativa aos 6 meses de tratamento, que resultou em um valor médio do IMC de 32,7. Ou seja, uma redução do IMC de cerca de 4\%. Conclusões: A intervenção de enfermagem personalizada e seguimento intensivo favorecem uma boa aceitação ou adesão ao programa e permitem observar mudanças no estilo de vida dos pacientes imprescindiveis para o controle da obesidade.

Palavras-chave: obesidade; educação para a saúde; enfermagem; velhice 


\section{Introducción}

La prevalencia de obesidad en los países occidentales se ha incrementado en las últimas décadas, hasta tal punto que representa el trastorno nutricional de mayor relevancia en nuestro medio. El Instituto Nacional de Estadística (INE) de España publicó recientemente la prevalencia de obesidad en la población mayor de 65 años de edad en ese país se estima en un 35\%. Aunque no es un problema exclusivo de los ancianos, este colectivo tiene especial vulnerabilidad, pues los problemas nutricionales son más frecuentes y los efectos más graves (1).

Por otro lado, datos actuales indican que los programas educativos aumentan los conocimientos o la sensibilización acerca de la enfermedad. Así, publicaciones hechas en países como Cuba y Brasil han reportado que la relación salud-educación debe ser una estrategia siempre exitosa, no solo para generar nuevos procesos de participación ciudadana en el desarrollo de estilos de vida saludables, sino también porque es importante para la reducción de riesgos de enfermar y morir. Por ello es primordial el apoyo de enfermería para que, con procesos de formación, los usuarios sean autónomos en la resolución de sus principales necesidades sanitarias (2).

El tratamiento de la obesidad es complejo; por ello hay que aplicar estrategias que incluyan, junto con las restricciones dietéticas pertinentes, actividades educativas que promuevan hábitos alimentarios y estilos de vida saludables. Son, entonces, fundamentales las medidas conductuales, para conocer y aumentar la motivación del paciente y ofrecerle estrategias que ayuden a manejar conductas erróneas relacionadas con la alimentación y con la actividad física, que es la mejor herramienta para evitar ganancias de peso (3).

La dieta constituye un pilar fundamental en el tratamiento de la obesidad. En los últimos cuarenta años los patrones de alimentación en España se han desviado progresivamente de una dieta saludable, y se ha podido apreciar la aparición de una enorme profusión de dietas heterodoxas, la mayoría de ellas basadas en modificaciones dietéticas totalmente alejadas de las recomendaciones de las guías clínicas (4).

Los ancianos constituyen un grupo importante de riesgo de malnutrición, condicionado por sus características fisiológicas, psicosociales, patológicas y farmacológicas. El peso excesivo está asociado al sedentarismo y a una progresiva reducción en los requerimientos calóricos para mantener el peso corporal (5).

La obesidad incrementa el riesgo de padecer enfermedades como infarto agudo de miocardio, accidente cerebro-vascular, hipertensión arterial, diabetes mellitus 2, osteoartrosis, várices, diferentes tipos de cáncer y demencia. Por ello es necesario profundizar en el conocimiento del problema y poner en marcha medidas de prevención, promoción y captación en las consultas de enfermería, que son muy frecuentadas por dicha población, para el control de sus patologías crónicas (1,3-4).

Enfermería tiene un papel fundamental (función docente) en la prevención y detección de diferentes problemas de salud, y la valoración del estado nutricional forma parte del cuidado habitual del anciano, tanto en 
las consultas de enfermería como en su propio domicilio. Incorporar la entrevista motivacional dentro de la terapia conductual contemplada en la intervención enfermera es importante, ya que la evidencia demuestra ser una estrategia de empoderamiento que genera un cambio positivo en los estilos de vida del paciente (6-9). Ante tal planteamiento, se realizó la investigación que presentamos a continuación:

\section{Objetivo}

Analizar los cambios en el indice de masa corporal (IMC) en un grupo de pacientes con obesidad, mediante una intervención enfermera intensiva basada en promover cambios en su estilo de vida mediante dieta hipocalórica, ejercicio físico y terapia conductual.

\section{Método}

Diseño del estudio: se realizó un estudio cuasi experimental (sin grupo control), con un muestreo no probabilístico, longitudinal y prospectivo.

Población: 212 pacientes usuarios de un centro de atención primaria de la provincia de Córdoba, España, que acuden a consulta de enfermería demandando alguna de las prestaciones que se ofertan en la cartera de servicios. Así se determinó el tamaño muestral considerando un error del 0,5, una proporción del 35\% de obesidad y un nivel de confianza del $95 \%$.

Criterios de inclusión: pacientes aparentemente sanos, con autonomía para las actividades de la vida diaria (AVD) y sin demencia senil (escala de Pfeiffer $<3-4$ errores).

Procedimiento: el seguimiento de los pacientes incluidos en el estudio se realizó en la consulta de enfermería de forma personalizada. En la primera visita se registraron las variables antropométricas en su historia clínica, junto con un estudio analítico general y hormonal, para descartar otras etiologías o comorbilidades, cuyo control puede llegar a adquirir prioridad frente a la pérdida de peso.

El estudio duró seis meses. En los dos primeros se realizó un seguimiento cada quince días, y en los cuatro meses restantes, una visita mensual. El objetivo de que estas consultas sean tan frecuentes es incrementar el control, la motivación del paciente y la adhesión al programa.

La recogida de información se dio por medio de una entrevista clínica con el paciente, que incluía un recopilatorio de datos sociodemográficos.

El programa basado en promover cambios en su estilo de vida, mediante dieta hipocalórica, ejercicio físico y terapia conductual, se realizó con recursos materiales (báscula de precisión, tallimetro, lipocalibre) y humanos (dietista, endocrino y enfermera), a fin de cumplir con estándares de calidad (validez/sensibilidad) establecidos para estos instrumentos y garantizar así la seguridad del paciente.

Dentro de la terapia conductual contemplada en la intervención enfermera se utilizó la entrevista motivacional, y para que el seguimiento fuese eficaz, se ha modificado el enfoque tradicional de felicitación/amonestación, dependiendo de si el paciente pierde o no peso, por el enfoque de refuerzo de hábitos/identificación y manejo de circunstancias adversas en los casos de buena 
o mala evolución, respectivamente. De esta forma, el paciente nos valora como un colaborador, no como a un juez a quien debe obedecer y rendir cuentas.

El programa incluye la disminución de la ingesta calórica, mediante el mantenimiento de una dieta equilibrada (mediterránea), la realización de ejercicio físico moderado y técnicas de terapia conductual, fundamentada en la entrevista motivacional (figura 1).

Figura 1. Organigrama del programa

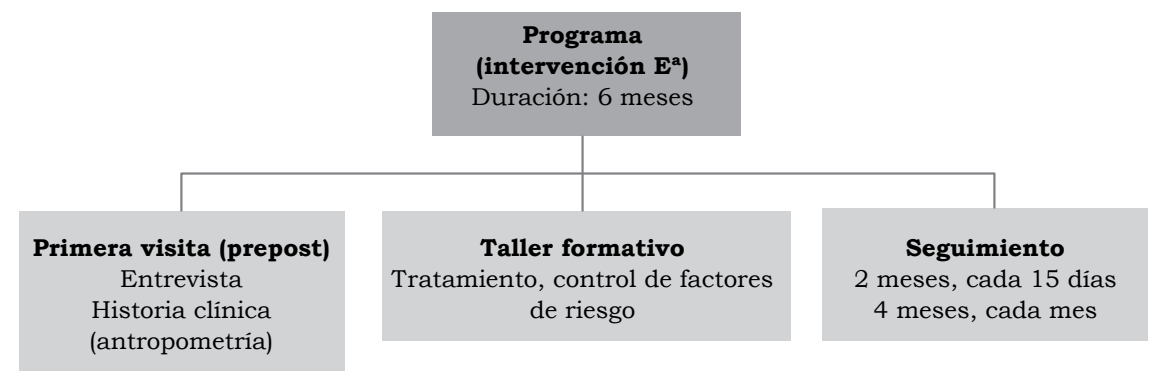

Fuente: resultados de investigación

Variables del estudio

Dependientes: parámetros antropométricos (peso e IMC).

Independiente: intervención terapéutica.

El IMC se calcula mediante la siguiente fórmula: peso (en kilogramos)/talla (en metros).

El valor más utilizado en clínica para determinar el estado nutricional de una persona o una población es el IMC, que es una medida de peso relativo que establece una relación entre la talla del sujeto y su peso. El IMC es un indicador no solo de la existencia de obesidad, sobrepeso o desnutrición, sino también del grado en que se padece.

Debido a la pérdida gradual de estatura en el anciano, los senescentes pesan más por unidad de altura que los adultos más jóvenes; en consecuencia, el IMC, que en adultos es normal si oscila entre 18,5 y 24,9 , en ancianos puede considerarse normal un IMC entre 22 y 26,9.

\section{Análisis estadístico}

Para este estudio nos hemos ayudado del programa de análisis estadístico Alcest (versión 1.5):

- El valor medio del IMC basal al inicio del estudio es de 34,2 (intervalo: 30,4-39,1).

- $\quad$ El valor medio del IMC en el subgrupo de mujeres es de 34,5.

- $\quad$ El valor medio del IMC en el subgrupo de hombres es de 33,9. 
De acuerdo con los datos previos, se calcula que la desviación típica del IMC en este tipo de pacientes es del orden de 2,3. Para un IMC inicial en el entorno de 30, una disminución del $4 \%$ corresponde a reducir dicho valor en 1,2. Con esos datos se predetermina que será necesario un tamaño de muestra del orden de unos 32 pacientes, para un contraste bilateral.

Pérdidas durante el estudio: un hombre falleció durante el estudio (por complicación de su patología) y otro no acudió a la última revisión por problemas familiares.

Mediante la prueba $\mathrm{T}$ de Student para muestras pareadas comparamos las medias del IMC antes de la intervención enfermera y tras esta, y observamos un descenso medio en el IMC de 1,4 (contraste estadísticamente significativo con $p<0,001)$.

Hallamos un tamaño de muestra escaso para permitir estudiar la existencia de asociación entre el descenso del IMC y otros factores, como podían ser sexo o edad del paciente. No obstante, se realizó un análisis de regresión múltiple, y como variable dependiente, la diferencia entre el valor final del IMC y el valor inicial, y variables independientes (sexo y edad), donde ninguno de los dos factores tuvo significación estadística.

Consideraciones éticas: siguiendo los principios éticos de la Declaración de Helsinki, los resultados obtenidos se emplearon exclusivamente para los fines de la investigación, y en ningún caso fueron reveladas las identidades de los participantes. Los datos obtenidos tuvieron un tratamiento estrictamente confidencial. Fueron aprobados por el Comité de Ética del Distrito (Córdoba). Todos los registros y su correspondiente consentimiento informado estuvieron debidamente custodiados por el investigador principal e identificados únicamente por el número asignado de caso, a fin de cumplir así con la legislación española sobre protección de datos (10).

\section{Resultados}

En la caracterización de nuestros pacientes, los sujetos del estudio estaban formados por una muestra de 32 personas con un IMC > 30: 16 hombres y 16 mujeres. Destaca que la media de edad se sitúa en torno a los 68 años (rango: 65-71). Un 93\% de ellos estaba casado; la media de hijos oscilaba entre $1,66-2$ y poseían 23,4 años de tiempo trabajado. Un 46,7\% se encontraba ya jubilado (tabla 1 ).

TABla 1. Características de la muestra estudiada

\begin{tabular}{|l|l|r|r|}
\hline \multicolumn{1}{|c|}{ Variable } & \multicolumn{2}{c|}{ Participantes (n = 32) } & \% \\
\hline Sexo & Varón-mujer & $16-16$ & $50-50$ \\
\hline Edad & Media (límites)-Moda & $68(65-71)-69$ & $\ldots$ \\
\hline Estado civil & Casado-otros & $30-2$ & $93,75-6,25$ \\
\hline Núm. hijos & Media-moda & $1,66-2$ & $28-13$ \\
\hline Tiempo cotización & Media-moda & $23,4-36$ & 100 \\
\hline Jubilados & Sí-No & $15-17$ & $46,67-53,33$ \\
\hline
\end{tabular}

FUENTE: elaboración propia 
El valor medio del IMC al final del estudio fue de 32,7 (intervalo: 28,2$38,7)$. Tras la intervención enfermera se ha producido (tabla 2):

- $\quad$ Reducción del IMC $\geq 5 \%$ en el 36,7\% de los pacientes (11).

- $\quad$ Reducción del IMC $\geq 4 \%$ en el 63,7\% de los pacientes (19).

- Únicamente 2 pacientes incrementaron su IMC durante el estudio, que suponen un 6,7\% del total.

Los factores de riesgo incluyeron, además del sobrepeso, ser fumador y sedentarismo al inicio del programa. De estos últimos, el $43 \%$ incorporó la actividad física tras finalizar el programa. El $24 \%$ era fumador, y de ello un $23 \%$ de los participantes abandonaron el hábito de consumo.

TABLA 2. Resultados de la investigación

\begin{tabular}{|l|l|}
\hline \multirow{4}{*}{ İtems } & Puntuación media \pm DE T-Student $(\mathrm{n}=30)$ \\
\cline { 2 - 2 } & IMC Media $(\mathrm{x})$. Inicio $34,2(30,4-39,1)$ \\
\cline { 2 - 2 } Mujeres & Final: 32,$7 ; \mathrm{p}=0,001$ \\
\hline \multirow{3}{*}{ Varones } & Inicio: 34,5 \\
\cline { 2 - 2 } & Final: $33,1 \pm 1.4 ; \mathrm{p}=0,001$ \\
\hline \multirow{2}{*}{ Percepción salud general } & Inicio: 33,9 \\
\cline { 2 - 2 } & Final: $32,5 \pm 1,4 ; \mathrm{p}=0,001$ \\
\cline { 2 - 2 } & Inicio: $55,5 \% ; \mathrm{p}<0,001$ \\
\hline
\end{tabular}

Fuente: elaboración propia

\section{Discusión}

El valor medio del IMC basal era de 34,2 al comienzo del estudio. Tras la intervención enfermera existe una progresiva disminución, que es estadísticamente significativa a los seis meses de tratamiento, pues resulta un valor medio en el IMC de 32,7 y constituye una reducción del IMC en torno al 4\%. Hay que tener en cuenta que los objetivos terapéuticos del programa no se han centrado en conseguir el peso ideal, sino que se han dirigido a conseguir pequeñas pérdidas de peso. Tales pérdidas han inducido una mejoría significativa en las comorbilidades asociadas de los pacientes tratados. La totalidad de los pacientes tratados refieren que se encuentran mejor animicamente y con mayor capacidad funcional y manifiestan que van a seguir acudiendo a consulta de enfermería para controlar su peso (55\% vs. $78 \%$ ).

En 2006, Miller propuso la definición actual de entrevista motivacional: "Proceso narrativo que evoca en el paciente razones para el cambio y compromiso para cambiar". Se caracteriza por ser cooperativa, respetuosa y evocativa; de ahí nuestra pretensión: exteriorizar algo positivo en los pacientes para activar su propia motivación $(6,7)$.

Anteriores estudios han avalado la intervención enfermera como una medida muy útil para el afrontamiento de la obesidad en cualquier etapa de 
la vida (11-17). En muchas ocasiones, enfermería es el escalafón sanitario más accesible y más cercano a la población, situación que debemos aprovechar para realizar la captación y el tratamiento del paciente obeso.

Hoy en día existe evidencia epidemiológica para considerar la obesidad como factor de riesgo para el desarrollo de enfermedades crónicas de gran prevalencia e incidencia en las sociedades actuales (18-22). En este sentido, el análisis de la Cochrane Collaboration (16) recomienda que en el diseño de nuevas intervenciones en salud pública se potencien aspectos relacionados con:

- $\quad$ Entornos que apoyen y faciliten cambios de conducta (dietas más saludables).

- Mejora en la oferta de alimentos en los centros geriátricos y escolares.

- Apoyo a las familias (modificar sus complejas circunstancias de vida y trabajo).

- $\quad$ Estudios de seguimiento (a largo plazo) que midan resultados más destacados y que incluyan evaluaciones económicas.

Una intervención efectiva que reduzca peso disminuye el riesgo de padecer estas enfermedades, por lo que fomentamos la seguridad del paciente. Además, está demostrado que pequeñas pérdidas de peso, en torno al 4\%-5\%, son capaces de mejorar espectacularmente las comorbilidades asociadas $(1,23-25)$.

Como se conocian las características de cada paciente por su historia clínica, se recomendó un tratamiento nutricional basado en la evidencia, individualizado, seguro y consensuado con el médico en cada caso, ya que hay que tener en cuenta que la selección de una dieta inadecuada, no solo podría ser ineficaz en alcanzar el objetivo de pérdida de peso, sino que podría acarrear toda una serie de consecuencias adversas, como son: originar situaciones de desnutrición o déficit de diferentes tipos de macronutrientes, favorecer el desarrollo de trastornos de la conducta alimentaria (de enorme gravedad y peor pronóstico que la propia obesidad) o fomentar sentimientos de frustración que afectan negativamente su estado psicológico $(26,27)$.

Son incontables y muy populares las dietas milagro que han proliferado para el tratamiento de la obesidad, algunas de ellas con una apariencia seudocientífica $(5,13,23)$. Estas dietas constituyen en la mayoría de los casos no solo un fraude, sino también un peligro para la salud, ya que se caracterizan por ser desequilibradas e insuficientes, prohibir alimentos básicos, ser monótonas y promover el ayuno. A largo plazo, se retomarán los hábitos anteriores y se producirá un efecto rebote que hará cada vez más difícil adelgazar. Por todo ello, nuestra propuesta dietética es una dieta equilibrada, completamente alejada de dietas restrictivas, acompañada de ejercicio físico y terapia conductual $(6,8)$.

\section{Conclusiones}

La intervención enfermera personalizada y el seguimiento intensivo favorecen una buena aceptación o adhesión al programa y permiten observar cambios en el estilo de vida de los pacientes, imprescindibles para el control de la obesidad. 


\section{Recomendaciones}

1. Explorar qué tipo de intervenciones enfermeras favorecen la adhesión y aceptación de nuestras propuestas a los pacientes.

2. Realizar este estudio con un tamaño muestral mayor y rango de edad más amplio para lograr mayor precisión en los resultados.

\section{Limitaciones}

El tiempo con el que se ha contado para el estudio ha sido escaso para determinar si las medidas propuestas pueden ser realizables por un espacio de tiempo prolongado o si previene futuras ganancias de peso. Por otro lado, destacar que el limitado tamaño muestral estudiado puede dificultar la extrapolación de resultados a poblaciones diferentes y debe ser considerado (establecer asociación entre el descenso del IMC y otros factores como el sexo o la edad del paciente).

\section{Financiación}

Ninguna

\section{Conflicto de interés}

Ninguno

\section{Referencias}

1. Gargallo M, Basulto J, Breton I, Quiles J, Formiguera X et al. Recomendaciones nutricionales basadas en la evidencia para la prevención y el tratamiento del sobrepeso y la obesidad en adultos. Nutr Hosp. 2012;27(3).

2. Arteaga C, Kolling M, Mesquida P. Health and education: A binomial worth being rescued. Rev Bras Educ Med. 2007;31:60-6.

3. Varela G, Requejo AM, Ortega RM, Zamora S, Salas J, Cabrerizo L et al. Asociación de enfermeras de nutrición y dietética [internet]. 2013. Disponible en: http://www.fesnad.org/pdf/Libro_Blanco_FEN_2013.pdf

4. Encuesta Nacional de Ingesta Dietética Española (AESAN) [internet]. 2011. Disponible en: http://www.aesan.msc.es/AESAN/web/notas_ prensa/presentacion_enide.shtml

5. De Torres ML, Francés M, Martínez JR. La dieta equilibrada: guía para enfermeras de atención primaria [internet]. Madrid: Sociedad Española de Dietética y Ciencias de la Alimentación (Sedca); 2007. Disponible en: http://www.nutricion.org/publicaciones/pdf/Gu \%C3\%ADa \%20 AP-Diet \%C3\%A9ticaWeb.pdf

6. Rollnick S, Miller WR, Butler CC. Motivational Interviewing in health care: Helping patients change behavior. New York: Guilford Press; 2008.

7. Sánchez J. Entrevista motivacional en atención primaria. E-Drogas [internet]. 2009. Disponible em: http://www.e-drogas.es/documents/10156/ cdcbf696-1abd-4161-94d1-9b1cb90c9656

8. Rivera S, Villouta F, Ilabaca A. Entrevista motivacional: ¿cuál es su efectividad en problemas prevalentes de la atención primaria? Aten 
Prim [internet]. 2008;40(5). Disponible en: http://www.elsevier.es/esrevista-atencion-primaria-27-articulo-entrevista-motivacional-cuales-su-13120020

9. Cubero J, Calderón M, Costillo E, Ruiz C. La educación para la salud en el espacio europeo de educación Superior [internet]. Granada: Universidad de Granada; 2011. p. 51-63. Disponible en: http://digibug.ugr.es/bitstream/10481/24743/1/Articulo\%203\%20publicaciones \%2041.pdf

10. Task Force US Preventive Services. Screening and interventions for overweight in children and adolescents: recommendation statement. Pediatrics. 2005;116(1):205-9.

11. Alba R. Estudio sobre prevalencia de obesidad infantil en niños escolarizados en un centro de Córdoba. Rev Educ Hekademos [internet]. 2014;15:65-71. Disponible en: http://www.hekademos.com/hekademos/media/articulos/15/06.pdf

12. Junta de Andalucía, Consejería de Salud. Plan para la Promoción de La Actividad Física y la Alimentación Equilibrada 2004-2008 (Pafae) [internet]. 2012. Disponible en: http://www.juntadeandalucia.es/salud/sites/csalud/galerias/documentos/c_1_c_6_planes_estrategias/ plan_alimentacion_equilibrada/Plan_actividad_fisica.pdf

13. Ley Orgánica 15/1999 de 13 de diciembre, sobre protección de datos de carácter personal. España; 1999.

14. Whitlock EP, Williams SB, Gold R, Smith PR, Shipman SA. Screening and interventions for childhood overweight: a summary of evidence for the US Preventive Services Task Force. Pediatrics. 2005;116(1):125-44.

15. Sumerbell C, Waters E, Edmunds L, Kelly S, Brown T, Campbell K. Interventions for preventing obesity in children. Cochrane Database Systet Rev. 2005;(3):CD001871.

16. Aranceta J. Situación actual de la alimentación en España. En: Guías alimentarias para la población española. Madrid: SENC; 2001.

17. Centers for Disease Control. Overweight and obesity [internet]. 2004. Disponible en: http://www.cdc.gov/obesity/index.html

18. Organización Mundial de la Salud. Conjunto de recomendaciones sobre la promoción de los alimentos y bebidas no alcohólicas dirigida a los niños. Ginebra: OMS; 2010.

19. Aranceta J, Pérez Rodrigo C, Serra Majem LL. Prevalencia de la obesidad en España: resultados del estudio SEEDO 2000. Med Clin. 2003;120:608-12.

20. Vásquez F, Díaz E, Lera L. Estudio longitudinal de la composición corporal por diferentes métodos como producto de una intervención integral para tratar la obesidad en escolares chilenos. Nutr Hosp. 2013;28(1). http://dx.doi.org/10.3305/nh.2013.28.1.6149

21. Morales P, JL Santos, A González. Validación factorial de un cuestionario para medir la conducta de comer en ausencia de hambre y su asociación con obesidad infantil. Rev Chil Pediatr. 2012;83(5):431-7.

22. Holmboe-Ottesen $\mathrm{G}$, Wandel $\mathrm{M}$. Changes in dietary habits after migration and consequences for health: a focus on South Asians in 
Europe. Food Nutr Res. 2012;56:18891. doi: http://dx.doi.org/10.3402/ fnr.v56i0.18891

23. Encuesta Europea de Salud en España (INE) [internet]. 2009. Disponible en: http://www.ine.es/jaxi/menu.do? type=pcaxis $\&$ path $=\% 2 \mathrm{Ft} 15 /$ $\mathrm{p} 420 \&$ file $=$ inebase $\& \mathrm{~L}=0$

24. World Health Organization. Recomendaciones mundiales sobre actividad física para la salud. Geneva: WHO Library Cataloguing-inPublication Data; 2010.

25. De Lago M. Spanish move away from Mediterranean diet. BMJ. 2011; 342: 1509. 11.

26. González E, Merino B. Guía Nutrición saludable y prevención de los trastornos alimentarios [internet]. 2000. Disponible en: http://www. fhspereclaver.org/userfiles/file/guia_nutricion_saludable.pdf

27. Pichot P. Manual diagnóstico y estadístico de los trastornos mentales DSM IV-TR. 4ª ed. Madrid: Masson; 2009. 